\title{
ESTRATEGIA COMPETITIVA Y RENTABILIDAD DE LAS EMPRESAS PERUANAS EXPORTADORAS DE POLOS DE ALGODÓN
}

\author{
COMPETITIVE STRATEGY AND PROFITABILITY OF PERUVIAN \\ COMPANIES THAT EXPORT COTTON T-SHIRTS
}

Carol Rocío Lara Hidalgo Universidad Nacional Mayor de San Marcos Lima, Perú Correo electrónico: carasoy@gmail.com ORCID: http://orcid.org/0000-0002-4823-1671

\section{RESUMEN}

Objetivo: Inferir los elementos de la estrategia competitiva que afectan la rentabilidad de las empresas peruanas exportadoras de polos de algodón. Método: El método de la investigación es correlacional al tratar de identificar y formular relaciones existentes entre las variables propuestas en base a datos históricos y datos obtenidos de 144 empresas exportadoras peruanas de polos de algodón. Para el análisis y procesamiento de datos se utilizaron técnicas de correlación lineal simple, Pearson, Spearman y Chi Cuadrado. Los programas usados fueron Microsoft Excel e IBM SPSS. Se usó información primaria (encuesta y estados financieros) y secundaria (estadística gubernamental, tesis y artículos científicos). Resultados: Se determinó que existe correlación directa entre costos y gastos con ventas de las empresas, este mismo caso se presenta entre exportaciones nacionales y ROE, asimismo, se evidencia relación directa entre los precios de los polos de algodón y exportaciones. El diseño es otro componente que tiene un impacto directo en la rentabilidad de la empresa. Conclusiones: Existe una estrecha relación entre los indicadores por el uso de la estrategia de liderazgo en costos y la estrategia de diferenciación que influyen en la rentabilidad de las empresas exportadoras.

Palabras clave: Competitividad; estrategia; costos; diferenciación; polos; rentabilidad.

\begin{abstract}
Objective: Infer the elements of the competitive strategy that affect the profitability of the cotton t-shirt Peruvian exporting companies. Method: The research method is correlational when trying to identify and formulate existing relationships between the proposed variables based on historical data and obtained data from surveys of 144 cotton t-shirt Peruvian exporting companies. For the analysis and processing data, simple linear correlation techniques, Pearson, Spearman and Chi Squared were used. The Programs Microsoft Excel e IBM SPSS used were. Primary (survey and financial statements) and second (government statistics, thesis and scientific articles) information was used. Results: It determine that exists a direct correlation between costs and expenses with company sales, this same case presents between the national exports and ROE, likewise, it evidence a direct relationship between cotton t-shirt prices and exports. The design is other component that has a direct impact on the profitability of the company. Conclusions: Exist a close relationship between indicators by the use of the Strategy of Leadership in Costs and Strategy of Differentiation that influence the profitability in exporting companies.
\end{abstract}

Keywords: Competitiveness; strategy; costs; differentiation; t-shirts; profitability. 


\section{INTRODUCCIÓN}

El comercio internacional está en un proceso de importante desarrollo. Teorías científicas surgidas en siglos pasados que marcaron la pauta de la dinámica mundial por mucho tiempo se contrastan con el nuevo escenario comercial y se hacen evidentes las limitaciones o la validez de las mismas.

Desde la teoría de la ventaja absoluta, según la cual el superávit comercial de un país no tenía por qué suponer un déficit comercial para otro, sino que esto permitiría el intercambio comercial beneficioso para ambos países (Czinkota y Ronkainen, 2014); pasando por la teoría de la ventaja comparativa que sostiene que los países tienden a especializarse en la producción y exportación de bienes que fabrican con un costo más bajo, llegamos a la nueva teoría del comercio que "son reducciones de los costos unitarios que se obtienen con grandes volúmenes de producción" (Hill, 2011, p.169). En el comercio surge lo que Feenstra, Taylor y Sancho (2011) señalan: "La externalización es la provisión de un servicio o la producción de diversas partes de un bien en distintos países, que posteriormente se emplean o se montan en un bien final de otro país" (p. 228).

La apertura de los mercados es la tendencia que predomina y esto genera mayor competitividad entre naciones y empresas. El principal producto de exportación de prendas de vestir son los polos de algodón (t-shirts) cuyo volumen de ventas no está tendiendo un crecimiento sostenido en los últimos años.

El subsector confecciones se encarga de la producción de polos de algodón y es el que maneja la oferta de productos textiles no tradicionales, cuyo crecimiento beneficia a la competitividad nacional en mayor grado que los productos tradicionales. La competitividad del país requiere promover la industria con valor agregado basado en la institucionalidad, innovación y articulación productiva (García-Vega, 2010).

En el sector industrial peruano hay varios rubros o cadenas importantes como el textil-confecciones. El sector textil peruano cuenta con empresas exportadoras que, según Flores (2014) al usar el modelo clúster mejora su competitividad, su gestión administrativa y comercial para los negocios internacionales.

Según Ercilio (2013), los factores que propiciaron el despegue de las exportaciones nacionales de prendas fueron las prácticas discriminatorias e incumplimiento de los derechos laborales de los trabajadores entre 1978 y 2010, debido a las reformas de contratación laboral que permitieron mayores exportaciones a grandes empresas. Goytizolo, Molina, Noriega y Tello (2017) afirman que la política del gobierno y el escenario internacional determinan las exportaciones, en su estudio concluyen que las exportaciones de polos se vieron disminuidas entre los años 2011 al 2015, debido a la política de reducción de tasa de restitución drawback así como el incremento del salario mínimo vital en el país.

Respecto a la desventaja de las Pymes frente a las grandes empresas, Gutiérrez, Lockuan y Ura (2003) concluyen que las alianzas estratégicas son la vía para incrementar la competitividad. Si la empresa no puede ser competitiva, los grandes clientes internacionales buscarán otros proveedores en el extranjero, lo que Walsh (2008) denomina outsourcing. El outsourcing de prendas de vestir hecho por Estados Unidos (nuestro principal comprador de polos) se realizó en lugares próximos a ese país y luego se transfirió a lugares más distantes y potencialmente más costosos como Asia, esto sumado al fin del Acuerdo Multifibras, explica el progreso de las exportaciones de prendas asiáticas, frente a la reducción de la producción latinoamericana (Suiter, 2016). Esto no quiere decir que las empresas de países fuera de Asia no sean competitivas, ya que, según el estudio realizado por Mercado, Fontalvo y De la Hoz (2011) en el cual comparan empresas asiáticas y colombianas, la economía perfectamente competitiva del mercado chino marca la diferencia, sobre todo, con respecto al volumen de la producción, siendo así el factor determinante la economía de escala.

Sin embargo, Allen (2008) sostiene que las empresas deben desarrollar estrategias que les permitan competir con pares en todo el mundo, ya que independientemente del país que se trate características similares que se presentan en empresas grandes del sector confecciones.

Porter (2008) definió estrategia competitiva como un conjunto de acciones que permiten tener una posición ventajosa en el escenario competitivo en el que operan las empresas. Hay tres estrategias genéricas que las empresas pueden usar e incluyen: la estrategia de liderazgo en costos, estrategia de diferenciación y estrategia de enfoque. En este caso, consideramos solo las estrategias de liderazgo en costos y de diferenciación debido a la posibilidad de establecer sus indicadores. Las fuentes de liderazgo en costos son variadas y dependen de la estructura del sector industrial, pueden incluir economías de escala u acceso preferencial a materias primas. Los costos deben considerar no sólo la manufactura sino también los costos de mercadotecnia, servicio e infraestructura. La ausencia de un marco de trabajo sistemático para el análisis de costos en la mayoría de empresas enfatiza las dificultades que tienen para determinar sus propios precios. A pesar de la importancia de diferenciación, sus fuentes no son bien comprendidas y muchas empresas la consideran en términos de producto físico o en la mercadotecnia, en lugar de enfocarse en la cadena de valor. La diferenciación no es 
solo ofrecer un producto distinto o de mayor precio que el de la competencia. Se puede alcanzar con inversión en tecnología, sistemas de información, calidad, optimización en el tiempo de entrega, investigación y diseño. Además, estos deben aportar a la cadena de valor de la empresa $y$, por tanto, al producto final.

Rentabilidad es un concepto que se aplica a toda acción económica en la que se movilizan medios materiales, humanos y financieros con el fin de obtener resultados. En sentido general, se refiere a los beneficios económicos que producen los capitales utilizados en un período de tiempo; existen indicadores para determinar la rentabilidad tales como: Margen Neto, Rentabilidad sobre Activos (ROA), Rentabilidad sobre Capital (ROE), Valor Económico Agregado (VEA) entre otros.

\section{MATERIALES Y MÉTODOS}

La investigación desarrollada es de tipo correlacional porque se formulan las hipótesis identificando relaciones existentes entre variables propuestas y explicativa al tratar de generar un sentido de entendimiento. Asimismo, es transversal pues recoge y analiza datos de la rentabilidad de las empresas peruanas exportadoras de polos de algodón entre los años 2011-2018. La unidad de estudio está representada por empresas peruanas exportadoras de polos de algodón en calidad de activas. Al 2018, el número total de empresas debidamente registradas en el Sistema Nacional de Administración Tributaria (SUNAT), ascendía a 343, el tamaño de la muestra se determinó con una selección no aleatoria y se usó la fórmula de cálculo del tamaño muestral para pruebas no paramétricas y se trabajó con un nivel de confianza del $95 \%$, esto quiere decir que $\mathrm{Z} \alpha=1,96$. Reemplazando los valores en la siguiente fórmula, la muestra asciende a 144 empresas.

$$
n=\frac{N * Z_{a}^{2} p^{*} q}{d^{2} *(N-1)+Z_{a}^{2} p^{*} q}
$$

El proceso de recolección de datos se llevó a cabo usando una encuesta de 15 preguntas, la cual fue congruente con lo establecido en la operacionalización de las variables. La encuesta se sometió a valoración de un panel de expertos, quienes emitieron su opinión para la adecuación de las preguntas. Asimismo, para determinar el grado de confiabilidad en el instrumento de estudio, se aplicó el coeficiente de consistencia interna alfa de Cronbach ( $\alpha$ ), debido a que presenta diferentes tipos de escala dentro de sus reactivos (nominal y Likert), así obtener el valor de alfa de Cronbach mediante un modelo multivariado.

Para el análisis y procesamiento de datos se utilizaron técnicas de correlación lineal simple, Pearson, Spearman y
Chi Cuadrado. Los programas usados fueron Microsoft Excel e IBM y SPSS. Además, se recogió información de los estados financieros e información secundaria (estadística gubernamental, tesis y artículos científicos).

\section{RESULTADOS}

Para hallar una relación entre liderazgo en costos y rentabilidad, se usó la correlación de Pearson relacionando costos y gastos con ventas en valores monetarios que se extrajeron de los estados financieros de la empresa. Como el $\mathrm{P}$ valor $(0,000)$ fue menor que el nivel de significancia $(0,05)$, se concluye que existe una correlación directa entre costos y gastos con las ventas; es decir, que las ventas de la empresa ha sido influenciada por los gastos y costos incurridos en el periodo de análisis (ver tabla 1).

El liderazgo en costos se puede alcanzar con la producción en escala que a su vez requiere mayor volumen de ventas para obtener más rentabilidad. Se tomaron las exportaciones nacionales de polos de algodón en kilogramos y se relacionó con el ROE, un indicador de rentabilidad de la empresa Creditex.

Por ser ROE una variable cuantitativa continua, se realizó el análisis estadístico descriptivo y se comprobó que los datos no tienen una distribución normal, por eso se utilizó el análisis de Correlación de Spearman para determinar si existe una relación entre las variables; al contrastarse las exportaciones y la rentabilidad, se obtiene un $\mathrm{P}$ valor del 0,024 y como este resultado es menor que el nivel de significancia $(0,05)$, se afirma que la rentabilidad de la empresa Creditex en el periodo 2011-2018 ha sido influenciado por las exportaciones de este tipo de prendas a los diferentes mercados de destino.

A continuación, se analiza la relación entre exportaciones nacionales en valores FOB y los ingresos por ventas de la empresa Creditex en el período 2013-2018. Al contrastar las cifras se encontró una correlación directa de Pearson de 0,31 cuyo coeficiente de determinación fue 55,67\%. Estos indicadores prueban que existe una relación lineal débil pero directa entre ambas variables y además que las ventas de la empresa Creditex es explicada por las exportaciones en un $9,61 \%$, siendo influenciada por otras variables que no están dentro del modelo en un 90,39\%. En ambos casos se concluye que, las exportaciones influyen positivamente en las ventas de la empresa (ver tabla 3 ).

La diferenciación se basa en el valor que permite hacer diferente a un producto de otro y reflejarlo en el precio. Se analizó la relación del precio unitario por kilogramo $(\mathrm{kg})$ del polo exportado con las exportaciones de polos en valores monetarios desde el año 2010 para obtener la relación entre diferenciación y rentabilidad de las empresas. 
Tabla 1

Coeficiente de correlación Pearson: Costos - Gastos y Ventas de Empresa "A".

\begin{tabular}{llrr}
\hline & & $\begin{array}{c}\text { Costos y Gastos } \\
\text { Empresa A }\end{array}$ & $\begin{array}{c}\text { Ventas } \\
\text { Empresa A }\end{array}$ \\
\hline \multirow{2}{*}{ Costos y Gastos Empresa A } & Correlación de Pearson & 1 & $1,000^{* *}$ \\
& Sig. (bilateral) & 5 & 0,000 \\
& $\mathrm{~N}$ & $1,000^{* *}$ & 5 \\
\hline \multirow{2}{*}{ Ventas Empresa A } & Correlación de Pearson & 0,000 & 1 \\
& Sig. (bilateral) & 5 & 5 \\
\hline **. La correlación es significativa en el nivel 0,01 (2 colas). & & \\
\hline
\end{tabular}

Fuente: Estados financieros empresa “A”. Elaboración propia.

Tabla 2

Correlación entre Exportaciones nacionales en $\mathrm{kg}$ y ROE de Creditex.

\begin{tabular}{|c|c|c|c|c|}
\hline & & & $\begin{array}{c}\text { Exportaciones } \\
\text { nacionales en kg. }\end{array}$ & ROE de Creditex \\
\hline \multirow{6}{*}{ Rho de Spearman } & \multirow{3}{*}{$\begin{array}{l}\text { Exportaciones nacionales } \\
\text { en } \mathrm{kg}\end{array}$} & Coeficiente de correlación & 1,000 & 0,643 \\
\hline & & Sig. (bilateral) & . & 0,024 \\
\hline & & $\mathrm{N}$ & 12 & 12 \\
\hline & \multirow{3}{*}{ ROE de Creditex } & Coeficiente de correlación & $0,643^{*}$ & 1,000 \\
\hline & & Sig. (bilateral) & 0,024 & \\
\hline & & $\mathrm{N}$ & 12 & 12 \\
\hline
\end{tabular}

Fuente: Elaboración propia con datos de SIICEX. BVL.

Tabla 3

Coeficiente de correlación de Pearson: Exportaciones nacionales en valor FOB y ventas de Creditex en U\$S.

\begin{tabular}{lccc}
\hline & Valor FOB U\$S & Ventas U\$S & Coef. Pearson $(\mathbf{r})$ \\
\hline 2013 & 159593293 & 82020606 & Determinación $\left(\mathrm{r}^{\wedge} 2\right)$ \\
2014 & 144343585 & 88222993 & \\
2015 & 124588915 & 82613939 & \\
2016 & 130535579 & 77809697 & \\
2017 & 148728959 & 83702121 & \\
2018 & 138973116 & 77989394 & \\
\hline
\end{tabular}

Fuente: Elaboración propia con datos de SIICEX. BVL.

El coeficiente de correlación de Pearson de 0,56 indica que hay una relación positiva y moderada entre las variables. Esto probaría una relación entre la estrategia de diferenciación basada en el precio unitario por kilogramo de polo de algodón con las ventas que obtienen las empresas. Por el valor de $\mathrm{R}^{2}$ también se determina que el valor unitario de polos por kg explica en un 31,38\% las exportaciones nacionales de polos de algodón (ver tabla 4 y figura 1).

Se realizaron preguntas en la encuesta que definen el uso de acciones de diferenciación y su relación con la rentabilidad. Se realizó la prueba de Chi cuadrado para determinar la relación entre la existencia de un área de investigación y diseño (que corresponde a la diferenciación) y la rentabilidad de la empresa. Al hacer la prueba de Chi Cuadrado, el $\mathrm{P}$ valor encontrado fue menor al nivel de significancia (sig) que es del 0,05; por lo que se afirma que el área de investigación y diseño (que corresponde al uso de una estrategia de diferenciación) está relacionado con la rentabilidad de la empresa (ver tablas 5 y 6 ).

Se puede buscar la diferenciación con una marca propia, se relaciona la existencia de una marca propia con la rentabilidad en la empresa. Al hacer la prueba de hipótesis 
Tabla 4

Coeficiente de correlación entre Precio unitario por kg. de polo de algodón y Exportaciones en valores FOB en U\$S.

\begin{tabular}{lccc}
\hline & Valor FOB U\$D en millones & Valor unitario U\$D & \\
\hline 2010 & 158 & 36 & Coef. Pearson $(r)$ \\
2011 & 174 & 42 & Determinación $\left(r^{\wedge} 2\right)$ \\
2012 & 163 & 42 & $31 \%$ \\
2013 & 160 & 45 & 40 \\
2014 & 144 & 38 \\
2015 & 125 & 38 \\
2016 & 131 & 40 \\
2017 & 149 & 39 \\
2018 & 139 &
\end{tabular}

Fuente: Elaboración propia con datos del SIICEX.

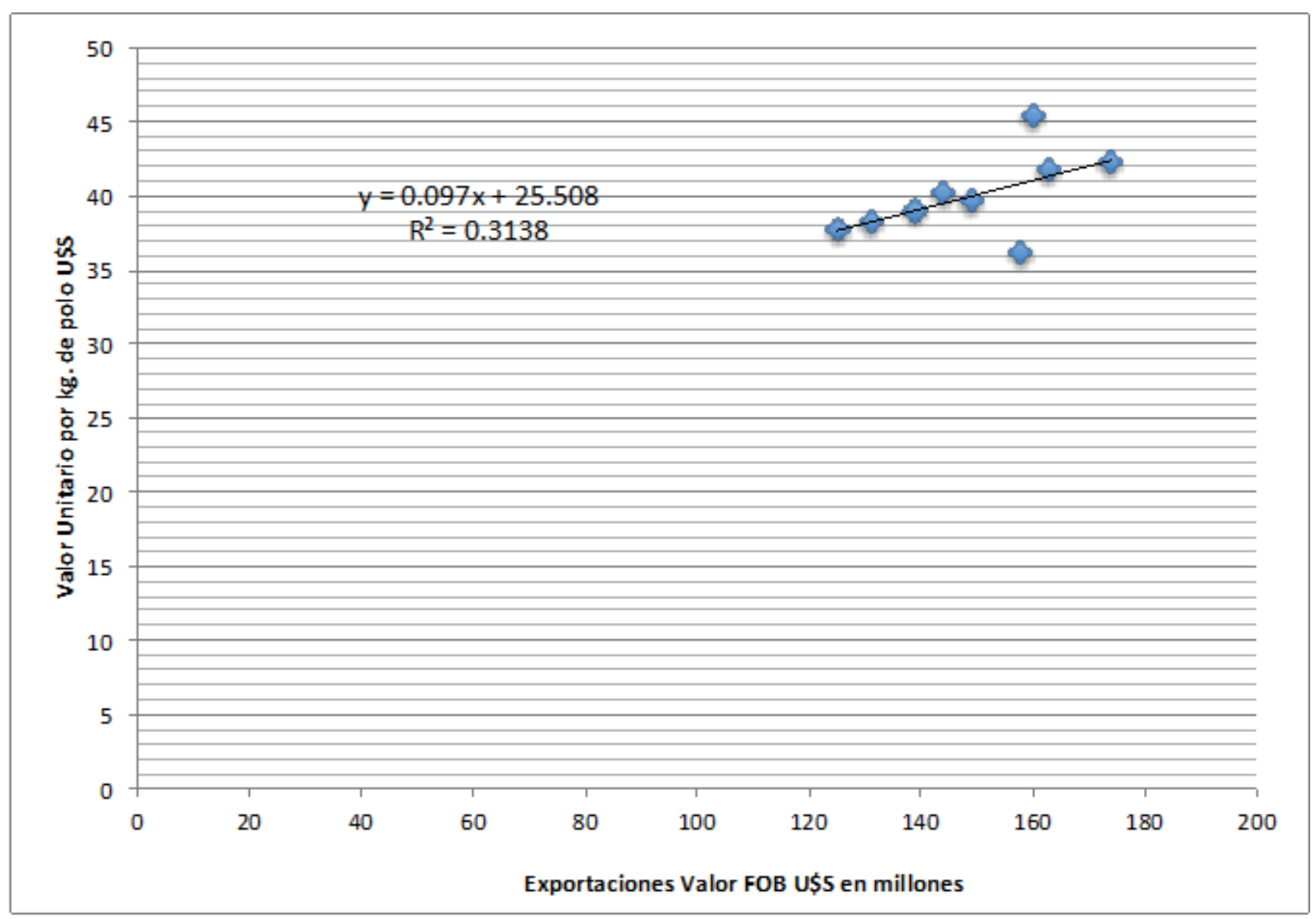

Figura 1. Correlación entre exportaciones y precio unitario por kg. de polo algodón.

Fuente: Elaboración propia con datos del SIICEX.

con la prueba Chi cuadrado, se encontró un $\mathrm{P}$ valor menor al margen de error permitido (sig) que es del 5\%, lo cual permite afirmar que, entre el uso de marca propia, que corresponde al uso de una estrategia de diferenciación y la rentabilidad de la empresa están relacionados; la empresa mejorará en sus beneficios si desarrolla o sigue fortaleciendo su propia marca (ver tablas 7 y 8 ).
Por haberse probado la relación entre indicadores que corresponden al liderazgo en costos (costos y gastos, exportaciones en kilogramos, exportaciones en valores monetarios) con indicadores de rentabilidad de las empresas (ventas y ROE), la estrategia de liderazgo en costos afecta la rentabilidad de las empresas peruanas exportadoras de polos de algodón. Se ha evidenciado 
Tabla 5

Tabulación cruzada: Investigación - Diseño y Rentabilidad.

\begin{tabular}{|c|c|c|c|c|c|}
\hline & & & \multicolumn{2}{|c|}{$\begin{array}{c}\text { ¿Cree Ud. que la investigación y } \\
\text { el diseño generan rentabilidad a la } \\
\text { empresa? }\end{array}$} & \multirow[b]{2}{*}{ Total } \\
\hline & & & No & Si & \\
\hline \multirow{6}{*}{$\begin{array}{l}\text { ¿Posee la empresa un área de investigación } \\
\text { y diseño }\end{array}$} & No & Recuento & 19 & 2 & 21 \\
\hline & & Recuento esperado & 2,8 & 18,2 & 21,0 \\
\hline & & $\%$ del total & $13,2 \%$ & $1,4 \%$ & $14,6 \%$ \\
\hline & $\mathrm{Si}$ & Recuento & 0 & 123 & 123 \\
\hline & & Recuento esperado & 16,2 & 106,8 & 123,0 \\
\hline & & $\%$ del total & $0,0 \%$ & $85,4 \%$ & $85,4 \%$ \\
\hline \multirow[t]{3}{*}{ Total } & & Recuento & 19 & 125 & 144 \\
\hline & & Recuento esperado & 19,0 & 125,0 & 144,0 \\
\hline & & $\%$ del total & $13,2 \%$ & $86,8 \%$ & $100,0 \%$ \\
\hline
\end{tabular}

Fuente: Elaboración propia con datos obtenidos de la encuesta.

Tabla 6

Prueba Chi cuadrado: Investigación - Diseño y Rentabilidad.

\begin{tabular}{llccc}
\hline & Valor & g.l. & $\begin{array}{c}\text { Sig. asintótica } \\
\text { (2 caras) }\end{array}$ & $\begin{array}{c}\text { Significación exacta } \\
\text { (2 caras) }\end{array}$ \\
\hline Chi-cuadrado de Pearson & $128,201^{\mathrm{a}}$ & 1 & 0,000 \\
Corrección de continuidad & 120,423 & 1 & 0,000 \\
Razón de verosimilitud & 99,130 & 1 & 0,000 & 0,000 \\
Prueba exacta de Fisher & & & & 0,000 \\
Asociación lineal por lineal & 127,311 & 1 & 0,000 & \\
N de casos válidos & 144 & &
\end{tabular}

a. 1 casillas $(20,0 \%)$ han esperado un recuento menor que 5. El recuento mínimo esperado es 2,77.

b. Sólo se ha calculado para una tabla $2 \times 2$

Fuente: Elaboración propia con datos obtenidos de la encuesta.

que contar con acceso a materia prima local y consolidar las ventas reducen los costos para las empresas peruanas.

Sin embargo, los costos de logística, mano de obra e incluso planta industrial tienden a elevar los costos de ventas $y$, por tanto, impactan en las ventas $y$ rentabilidad de las empresas peruanas. En el caso de la empresa analizada, se encontró que los gastos financieros afectan a los costos totales, confirmando la relación entre estos y la rentabilidad lograda a través de las ventas. En el caso de la gran empresa nacional, la estrategia debe darse para lograr economías de escala como señala Porter (2008), ya que eso permite reducir costos de producción y es la estrategia utilizada por los competidores internacionales. Al encontrarse relación entre los indicadores de una estrategia de diferenciación (precio unitario, investigación y diseño, marca propia) con rentabilidad de las empresas, se establece que la estrategia de diferenciación afecta a la rentabilidad de las empresas peruanas exportadoras de polos de algodón. La diferenciación que permite atribuir un mayor precio se basa, en el caso peruano, en la calidad del algodón.

\section{DISCUSIÓN}

Las empresas nacionales invierten en investigación de mercados extranjeros para la mejora de sus diseños, de acuerdo a los pedidos de los clientes que suelen tener marca propia. Especializarse en clientes industriales puede constituir una ventaja competitiva, como señala Porter (2008), pero también constituye una desventaja ya que al no vender productos terminados no se puede 
Tabla 7

Tabulación cruzada: Marca propia y Rentabilidad.

\begin{tabular}{|c|c|c|c|c|c|}
\hline & & & \multicolumn{2}{|c|}{$\begin{array}{c}\text { ¿Cree Ud. que la marca propia contribuye } \\
\text { a la rentabilidad de la empresa? }\end{array}$} & \multirow[b]{2}{*}{ Total } \\
\hline & & & No & Si & \\
\hline \multirow{6}{*}{$\begin{array}{l}\text { ¿La empresa tiene o está desarrollando } \\
\text { una marca propia de exportación? }\end{array}$} & No & Recuento & 95 & 0 & 95 \\
\hline & & Recuento esperado & 62,7 & 32,3 & 95,0 \\
\hline & & $\%$ del total & $66,0 \%$ & $0,0 \%$ & $66,0 \%$ \\
\hline & $\mathrm{Si}$ & Recuento & 0 & 49 & 49 \\
\hline & & Recuento esperado & 32,3 & 16,7 & 49,0 \\
\hline & & $\%$ del total & $0,0 \%$ & $34,0 \%$ & $34,0 \%$ \\
\hline \multirow[t]{3}{*}{ Total } & & Recuento & 95 & 49 & 144 \\
\hline & & Recuento esperado & 95,0 & 49,0 & 144,0 \\
\hline & & $\%$ del total & $66,0 \%$ & $34,0 \%$ & $100,0 \%$ \\
\hline
\end{tabular}

Fuente: Elaboración propia con datos obtenidos de la encuesta.

Tabla 8

Pruebas de chi-cuadrado: Marca propia y Rentabilidad.

\begin{tabular}{lcccc}
\hline & Valor & g.I. & $\begin{array}{c}\text { Sig. asintótica } \\
\text { (2 caras) }\end{array}$ & $\begin{array}{c}\text { Significación exacta } \\
\text { (2 caras) }\end{array}$ \\
\hline Chi-cuadrado de Pearson & $144,000^{\mathrm{a}}$ & 1 & 0,000 & \\
Corrección de continuidad ${ }^{\mathrm{b}}$ & 139,580 & 1 & 0,000 & \\
Razón de verosimilitud & 184,671 & 1 & 0,000 & 0,000 \\
Prueba exacta de Fisher & & & & 0,000 \\
Asociación lineal por lineal & 143,000 & 1 & 0,000 & \\
$\mathrm{~N}$ de casos válidos & 144 & & & \\
\hline
\end{tabular}

a. 0 casillas $(0,0 \%)$ han esperado un recuento menor que 5. El recuento mínimo esperado es 16,67.

b. Sólo se ha calculado para una tabla $2 \times 2$

Fuente: Elaboración propia con datos obtenidos de la encuesta.

llegar directamente al consumidor ni desarrollar productos sofisticados. De otro lado, Gutiérrez et al. (2003) infieren que las asociaciones necesarias para las empresas peruanas no se dan, pues muchas empresas manifestaron no tener ningún tipo de asociación con otras, lo cual constituye una desventaja frente a las asociaciones que practican las empresas asiáticas como sostienen Mercado et al (2011). Las certificaciones internacionales son también un aspecto que la mayoría de empresas nacionales no consideran para su competitividad y que no les permite diferenciarse pese a que invierten en investigación. Se concuerda con García-Vega (2010), ya que el sector confecciones (parte del sector textil peruano) necesita ser priorizado por ser uno de los sectores más importantes de la industria nacional, pero se necesitan mayores acciones por parte de las empresas para no perder su competitividad. Nuevos países han ingresado a competir en el mercado mundial, como sostiene Suiter (2016), gracias a estrategias competitivas e impulsados a la vez por las grandes empresas que han relocalizado sus plantas de manufactura como indica Walsh (2008) y que concuerda con lo que sostiene Hill (2011). Respecto a las nuevas tendencias del comercio internacional, esta viene afectando a trabajadores textiles en todo el mundo, incluyendo a los del Perú. Como señala Ercilio (2013), se han dado casos de explotación laboral para poder lograr el liderazgo en costos, aunque no es la mejor manera de lograr la competitividad, el sector confecciones peruano es nido constante de nuevas empresas que deben mantenerse con estas acciones para no dejar de ser rentables y desaparecer. Por los resultados encontrados, este estudio concluye que la estrategia de liderazgo en costos y la estrategia de diferenciación afectan a la rentabilidad de las empresas peruanas exportadoras de polos de algodón. 


\section{REFERENCIAS BIBLIOGRÁFICAS}

Allen, R. (2008). Performance Measurement of Textile and Apparel Supply Chains in Developing Countries (Tesis de Doctorado). University of North Carolina, Chapel Hill.

Czinkota, M., \& Ronkainen, I. (2014). Marketing Internacional. México D.F.: Cengage Learning.

Ercilio, F. (2013). Historia de las prácticas discriminatorias en las normas laborales: el caso de la industria de la exportación no tradicional en el Perú, 1978-2010 (Tesis de Doctorado). Universidad Nacional Mayor de San Marcos, Lima.

Flores, G. (2014). Modelo Empresarial Clúster En Negocios Internacionales Del Sector Exportador MYPES de Confecciones Textiles de Gamarra. Lima 2005 - 2012 (Tesis de Doctorado). Universidad Nacional Mayor de San Marcos, Lima.

Feenstra, R., Taylor, A. (2011). Comercio internacional. Barcelona: Editorial Reverté, S. A.

García-Vega, E. (2010) Competitividad en el Perú: Diagnóstico, sectores a priorizar y lineamientos a seguir para el período 2011-2016. Journal of Globalization, Competitiveness \& Governability. 5 (1), 112-141.

Goytizolo, A., Molina, E., Noriega, J., \& Tello, N. (2017). Causas que originaron que la industria textil peruana disminuya sus volumenes de exportación de la partida
61.09.10.00.00 hacia E.E.U.U entre los años 2011 y 2015 (Tesis para obtener el grado de Bachiller). Universidad Peruana de Ciencias Aplicadas, Lima.

Gutiérrez, E., Lockuan, A., \& Ura, L. (2003). Las alianzas estrategias como ventaja competitiva (Pyme: Sector Confecciones). (Tesis de Maestría). Universidad Peruana de Ciencias Aplicadas, Lima.

Hill, Ch. (2011). Negocios Internacionales: Competencia En el Mercado Global. New York: McGraw-Hill.

Mercado, H., Fontalvo, T., \& De la Hoz, E. (2011). Análisis Comparativo Entre Las Cadenas Productivas Del Sector Textil-Confecciones de La Provincia de Jiangsu-China y el Departamento Del Atlántico-Colombia. Ingeniare. Revista chilena de ingeniería. 19 (3), 429-441

Porter, M. (2008) Ventaja Competitiva. 15th ed. México D.F: Compañía Editorial Continental S.A. de C.V.

SIICEX. Sistema Integrado de Información de Comercio Exterior. http://siicex.gob.pe

Suiter, C. (2016). Competititon in the Post MFA World: Global Apparel Industry Reorganization and Quality Diferentiation. (Tesis de Maestría). University of $\mathrm{Ne}$ braska, Omaha.

Walsh, E. (2008). The Oursourcing of Apparel and Textiles: Manufacturing Site selection. (Tesis de Maestría). University of North Carolina, Greensboro. 